\title{
Indication of Highly Correlated Electron Transport in Disordered Multilayer Ferritin Structures
}

\author{
Chris Rourk*1a, Yunbo Huang ${ }^{2 a}$, Minjing Chen², Cai Shen*2 \\ 1. Independent researcher \\ 2. Ningbo Institute of Materials Technology \& Engineering Chinese Academy of Sciences. 1219 \\ Zhongguan Road, Zhenhai District, Ningbo, Zhejiang, China, 315201 \\ a: equal contribution \\ *E-mail: crourk@jw.com; shencai@nimte.ac.cn
}

\begin{abstract}
Tests of devices using a layer-by-layer deposition process for forming multilayer arrays of ferritin have been previously reported that indicate that highly-correlated electron transport is occurring, consistent with models of electron transport in quantum dots. This paper reports the results of the effect of various degrees of structural homogeneity on the function of these ferritin arrays, as well as demonstrating that these structures can provide a switching function associated with the circuit that they are contained within.
\end{abstract}

Keywords: Ferritin; Quantum dots; Layer-by-layer deposition; Conductive atomic force microscopy

\section{Introduction}

A quantum dot (QD) is a conducting island of a size comparable to the Fermi wavelength in all spatial directions. The current/voltage (I/V) characteristics of QDs have been previously studied using conductive atomic force microscopy (c-AFM), and it has been shown that QDs can exhibit nonlinear I/V behavior when tested. Unlike conventional linear I/V behavior of bulk materials that is a function of conductivity alone, the nonlinear I/V behavior of a QD is a function of whether the QD is behaving coherently or non-coherently [1]. This nonlinear behavior (either coherent or 
non-coherent) can be due to tunneling, which is an effect created by the wave-like characteristics of electrons. The measured electron tunneling distance associated with QDs is typically $10 \mathrm{~nm}$ but can be greater, and is a function of the size of the QD.

Ferritin is a spherical iron storage protein that is abundant in living organisms. C-AFM tests performed on ferritin cores deposited using a highly-disordered layer-by-layer deposition technique have demonstrated similar nonlinear I/V behavior as that seen in QDs, with a measured current of $0.4 \mathrm{nA}$ at $3 \mathrm{~V}$ over distances as great as $40 \mathrm{~nm}$, as well as anomalous current measurements of $0.4 \mu \mathrm{A}$ for disordered multilayer planar arrays of ferritin that have been deposited between parallel electrodes over distances as great as 40 microns, for an applied voltage differential between electrodes of up to $3 \mathrm{~V}$ [2-5]. A comparison of the c-AFM I/V response of a semiconductor QD and ferritin is shown in Fig. 1 in the Supporting Information, and shows that these I/V curves are similar. Notably, the c-AFM I/V response for both semiconductor QDs and ferritin exhibit 1) relatively flat current response at voltages up to 0.5 volts per QD or ferritin core, and 2) non-linear increases in current levels above a threshold voltage. In view of this c-AFM I/V behavior for individual ferritin cores, it would not be expected that a disordered multilayer planar array of ferritin would conduct electrons at an applied voltage differential of $3 \mathrm{~V}$, much less at a level that is three orders of magnitude greater than the current measured for individual cores over much shorter distances due to tunneling.

Electron transport through two-dimensional disordered QD arrays has been studied using computer models [6]. These computer models show that the electrical behavior of disordered QD arrays is different from the electrical behavior of individual QDs, and that electron transport occurs at much lower voltage levels due to incoherent tunneling between QDs. The modelled I/V characteristic for a number of different two-dimensional disordered arrays is log-linear above a relatively low threshold voltage, as shown in Fig. 3 in the Supporting Information. The model reported in [6] was based on QD arrays between parallel metal electrodes, where the QD arrays have a controllable degree of disorder that is modelled as variable inter-dot capacitances. The model was also based on a temperature of zero degrees, to allow the role of thermal fluctuations on tunneling rates to be ignored. Tunneling from metal electrodes to the QDs necessarily means that electrons are behaving as coherent or non-coherent waves when they tunnel, and they must also be exhibiting wavelike behavior in order to tunnel through the arrays. It appears that the tunneling behavior of electrons through disordered arrays of ferritin is thus similar to the tunneling 
behavior of electrons through disordered arrays of QDs. As shown in Fig. 4 in the Supporting Information, the current measured between two parallel metal electrodes having an interdigitated design was reported to have a log-linear/non-linear response.

Ferritin comprises a spherical protein shell that contains a complex internal structure that includes both ferrihydrite and ferrihydrite precursors [7], as shown in Fig. 2 in the Supporting Information. The protein shell includes light chain ferritin subunits that are able to conduct electrons into and out of the core [8]. Once an electron is inside of the core, the internal ferrihydrite structure is able to trap electrons for microsecond time scales [9]. In addition, the external protein structure of ferritin and the internal structure of the ferrihydrite and ferrihydrite precursors are both chiral, which can contribute to spin selectivity $[7,10]$ Chirality in organic structures, like the ferritin protein shell subunits, has also been observed to function as a spin filter, which would increase the spin coherence of exciton electrons generated by individual ferritin cores in disordered layers of ferritin cores [11]. Likewise, chirality of inorganic structures like the ferrihydrite core of ferritin has also been observed to facilitate interaction with chiral organic structures, like proteins [12]. Furthermore, it has been observed that mixed iron oxide phases are present in the ferritin core that include magnetite regions, which could also contribute to spin filtering [13]. These combined effects could help to explain the observed room-temperature electron transport of electrons through DMFS, which could create coherent or non-coherent tunneling at room temperature due to spin coherence that is similar to that modelled in the QD arrays at an assumed temperature of zero degrees $[14,15]$. This electron behavior is also consistent with the function of ferritin, which is to store and release iron as part of complex biological processes that utilize iron, because it would facilitate iron storage and release under suitable conditions (such as in response to the $\mathrm{pH}$ of the environment surrounding the ferritin core) [16].

The electron trapping function performed by ferrihydrite in the ferritin core involves the reduction of iron (III) to iron (II), which may be caused by defects in the crystalline structure of the ferrihydrite, or which may itself cause such defects to form. Because ferrihydrite precursors will be formed by the trapping of an electron by an iron (III) atom in the ferrihydrite crystalline structure and will be located in the immediate vicinity of the iron (II) atom that is subsequently formed, those compounds will be able to reform into the ferrihydrite crystalline structure if the iron (II) atom releases the trapped electron from the $3 \mathrm{~d}^{6}$ orbital. This process not only results in the release of an electron that has the same spin as other trapped electrons that may be released at 
or near the same time, but also causes the electron to lose energy with each trap and release event, which increases the de Broglie wavelength of the electron. The coherence length of the exciton (which contributes to the tunneling distance) is directly related to the de Broglie wavelength of the electron. This process could explain why ferritin is able to exhibit the same behavior as QD models at zero degrees, namely, because the role of thermal fluctuations on tunneling is not sufficient to overcome the effect of coherent spin and increased de Broglie wavelength of the electrons.

Ferritin may thus be able to provide a relatively inexpensive material with a low environmental impact for use in semiconductor devices. The layer-by-layer deposition technique would be useful for fabrication of semiconducting devices that use nanoparticles, which are otherwise very difficult to manipulate, and could possibly be used to reduce manufacturing costs for devices that use ferritin or even semiconductor QDs, if the process could be modified for use with other nanoparticles. Given the difficulty of both forming devices that use nanoparticles and of verifying the proper construction of such devices, a simple layer-by-layer process that creates repeatable and consistent results would be of potential value. However, the results reported in [14] do not provide much detail as to parametric measurements, including the I/V behavior of disordered ferritin layers for one through five layers, the I/V behavior for gaps of different sizes between electrodes and other important experimental details. Thus, the first objective of these tests was to determine whether the layer-by-layer process could provide consistent results that would allow for manufacture of commercial devices using the process.

It is also noted that highly correlated electron behavior has been reported in both QD arrays $[17,18]$ and twisted double bilayer graphene [19]. One of the observable characteristics of highly correlated electron behavior in twisted double bilayer graphene is collective electron behavior, such as changing from conductive to non-conductive. This highly correlated behavior could explain the conductivity of QD arrays, including disordered arrays, and would also predict that such arrays could be "switched" from blocking to conducting as a function of the design of the circuit containing the QD array, which was the second objective of these tests.

In order to determine whether electron transport that might occur in any DMFS that might be formed was due to either 1) quantum mechanical effects of coherent or non-coherent electrons or 2) classical electron conduction, a three electrode device was used. As shown below, the design is similar to that used in [14], but without the interdigitated structure. In addition, the interdigitated structure from [14] with 3 electrodes was also tested for comparison purposes. Ferritin was 
deposited using the layer-by-layer technique discussed in [14], and current was measured between the electrodes in 5 configurations:

1) with potential applied to electrode A1 and electrodes B1, B2 and B3 in series and connected to ground;

2) with potential applied to electrode A1, electrode B1 connected to ground and electrodes B2 and B3 floating;

3) with potential applied to electrode A1, electrode B2 connected to ground and electrodes B1 and B3 floating;

4) with potential applied to electrode A1, electrode B3 connected to ground and electrodes B1 and B2 floating; and

5) with potential applied to electrodes B1, B2 and B3 in parallel and with electrode A1 connected to ground. The voltage conventions used were based on electrodes B1-B3 being grounded and voltage being applied to electrode A1, such that when -3 volts was applied to electrodes B1-B3 in this configuration, it was recorded as +3 volts, and vice versus (i.e. the I/V curves are flipped about the $\mathrm{Y}$ axis).

(a)

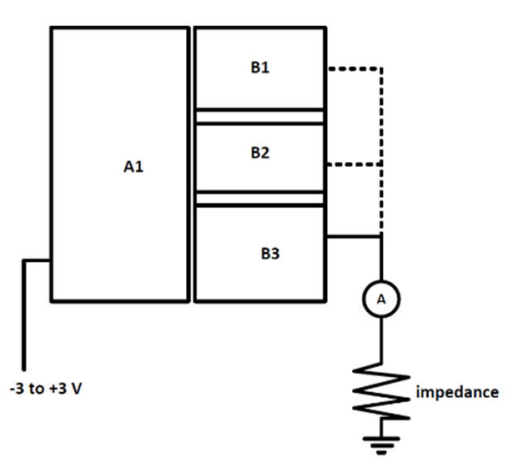

(c)

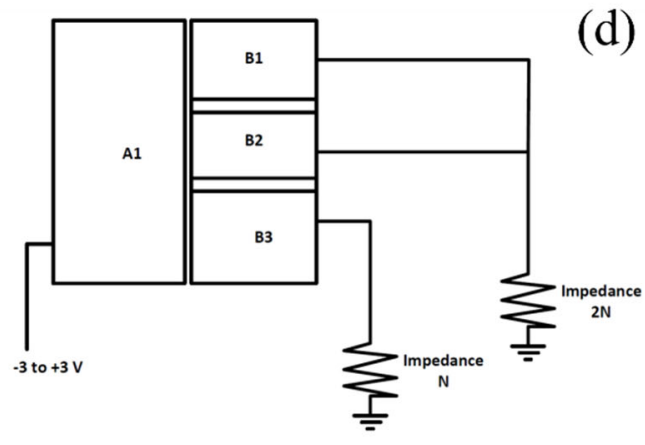

(b)

(d)
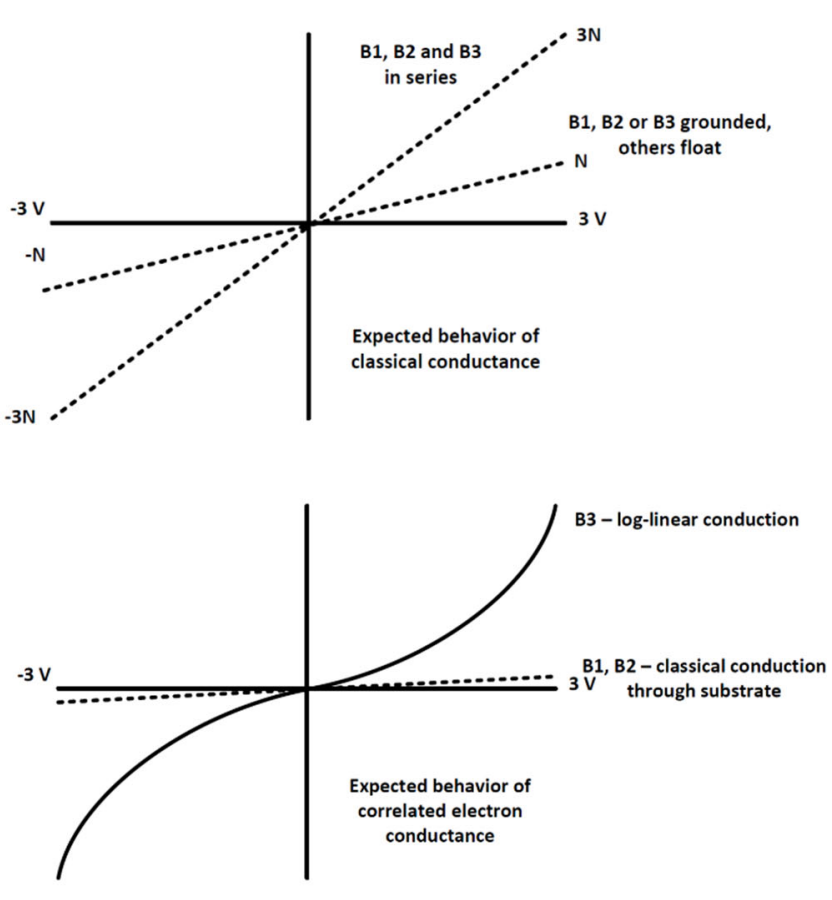

Fig. 1 - a) Circuit design, b) classical I/V current behavior, c) circuit design, d) coherent electron transport I/V behavior 
If the ferritin layers behave classically, then a linear I/V behavior as shown in Fig. 1(b) would be expected with one third of the current in each of the three electrodes B1, B2 and B3 as shown in the circuit of Fig. 1(a). However, if quantum mechanical electron transport occurs, then log-linear/non-linear I/V behavior would be expected, similar to what was seen in [6] and [14]. In addition, it would be expected that when two or more electrodes are connected to ground in parallel by the same impedance, that current flow would be blocked in either direction, because highly correlated electrons would need to flow through the same path or not at all. Otherwise, highly correlated electrons should follow the same lowest impedance path to ground, if multiple paths of different impedance were available. For example, the I/V behavior in Fig. 1(d) would be predicted for the circuit design in Fig. 1(c).

\section{Experimental Section}

\subsection{Ferritin deposition on Si surface}

Heavily doped n-type (As) silicon(111) wafers $(0.0025-0.004 \Omega \mathrm{cm}, 525 \mu \mathrm{m}$, SSP Prime from University Wafer) were cut into small $(10 \mathrm{~mm} \times 10 \mathrm{~mm})$ pieces, which were cleaned using ethyl acetate/acetone/ethanol (for $5 \mathrm{~min}$ in each). The solvent-cleaned wafer was placed in freshly prepared acid piranha solution $\left(\mathrm{H}_{2} \mathrm{SO}_{4}: \mathrm{H}_{2} \mathrm{O}_{2}=7: 3 \mathrm{v} / \mathrm{v}\right)$ for $10 \mathrm{~min}$ at $80^{\circ} \mathrm{C}$. The silicon wafer was washed thoroughly with excess deionized water and dipped in $2 \% \mathrm{HF}$ solution for $1 \mathrm{~min}$. The HFtreated hydrophobic silicon wafer was washed with deionized water, and then it was placed in freshly prepared base piranha solution $\left(\mathrm{NH}_{4} \mathrm{OH}: \mathrm{H}_{2} \mathrm{O}_{2}: \mathrm{H}_{2} \mathrm{O}=1: 1: 5 \mathrm{v} / \mathrm{v}\right)$ for less than 1 min at 70 ${ }^{\circ} \mathrm{C}$ to make the wafer surface hydrophilic via oxide layer formation and to remove the metallic contamination on the wafer surface. The wafer was finally rinsed with deionized water and dried under nitrogen gas.

Using the process discussed in [14], native equine spleen holoferritin (HoSF) (SigmaAldrich) was diluted to $200 \mathrm{nM}$ concentration using the required amount of MOPS-NaCl buffer (10 mM MOPS (Sigma) in $0.15 \mathrm{M} \mathrm{NaCl}$ (Merck) solution, $\mathrm{pH}$ 6.1). The diluted protein solution in MOPS- $\mathrm{NaCl}$ buffer was filtered using a $0.22 \mu \mathrm{m}$ syringe filter and the filtrate was stored at 4 ${ }^{\circ} \mathrm{C}$.

Cationized equine spleen holoferritin (HoSF) (Sigma-Aldrich) was diluted to $200 \mathrm{nM}$ concentration using the required amount of MOPS-NaCl buffer (10 mM MOPS (Sigma) in 0.15 
$\mathrm{M} \mathrm{NaCl}$ (Merck) solution, $\mathrm{pH}$ 6.1). The diluted protein solution in MOPS- $\mathrm{NaCl}$ buffer was filtered using a $0.22 \mu \mathrm{m}$ syringe filter and the filtrate was stored at $4^{\circ} \mathrm{C}$.

For protein monolayer formation, the negatively charged silicon substrate was incubated in $200 \mathrm{nM}$ cationized ferritin in MOPS-NaCl buffer for $15 \mathrm{~min}$. The protein-coated silicon surface was rinsed with deionized water, and then dried under nitrogen gas. For bi-layer formation, the cationized ferritin-coated silicon was incubated in $200 \mathrm{nM}$ holoferritin in MOPS-NaCl buffer for $15 \mathrm{~min}$. Then the protein-coated surface was rinsed thoroughly with deionized water, and dried under nitrogen gas. For further layer formation, the above-mentioned steps (coating with cationized ferritin and native ferritin) were repeated up to the desired number of protein layers. Samples deposited with ferritin are stored at $4^{\circ} \mathrm{C}$ in a nitrogen environment.

\subsection{Ferritin deposition on patterned $\mathrm{SiO}_{2} / \mathrm{Si}$ surface}

Also using the process discussed in [14], an electrode chip $(1 \mathrm{~cm} \times 1 \mathrm{~cm})$ composed of a silicon substrate, a silicon dioxide surface and a gold surface coating was fabricated for protein modification. First the chip was cleaned with soap water, followed by vigorous washing with deionized water. Then the chip was dried under hot air. Then the chip was treated with acetone at $50^{\circ} \mathrm{C}$ for $10 \mathrm{~min}$, then was treated with $5 \mathrm{~min}$ isopropanol at $70^{\circ} \mathrm{C}$, and washed with ethanol and deionized water. Finally, the chip was dried under nitrogen gas, and then it was ready for protein modification. Similarly the layer-by-layer protein deposition method is used to form DMFS on the electrode chip. Chips deposited with ferritin are stored at $4^{\circ} \mathrm{C}$ in a nitrogen environment.

\subsection{AFM and c-AFM Measurements}

AFM (Bruker Icon) and c-AFM (Bruker Icon) was carried out in a glovebox (MBRAUN, $\mathrm{H}_{2} \mathrm{O} \leqslant 0.1 \mathrm{ppm}, \mathrm{O}_{2} \leqslant 0.1 \mathrm{ppm}$ ). Topography and current of ferritin were simultaneously collected using the contact mode (PFTUNA tip, Bruker Corporation, Tip radius $25 \mathrm{~nm}$, Frequency $70 \mathrm{kHz}$, Spring constant $0.4 \mathrm{~N} / \mathrm{m}$ ). The image scan rate was set at $1 \mathrm{~Hz}$ per line with a resolution of $256 \times$ 256 pixels, corresponding to a frame rate of $256 \mathrm{sec}$ per frame.

\subsection{Lateral current measurements}

Electrodes were probed using a station with four tungsten tips with $10 \mu \mathrm{m}$ diameter by a Keithley semiconductor characterization system (Tektronix company, 4200-SCS). We apply -3 3 $\mathrm{V}$ voltage to electrode chips to measure lateral current.

\begin{tabular}{|l|l|}
\hline Method & Connection \\
\hline 1 & voltage applied to A1, B1-B3 grounded \\
\hline
\end{tabular}




\begin{tabular}{|l|l|}
\hline 2 & voltage applied to A1, B1 grounded, B2, B3 float \\
\hline 3 & voltage applied to A1, B2 grounded, B1, B3 float \\
\hline 4 & voltage applied to A1, B3 grounded, B1, B2 float \\
\hline 5 & voltage applied to B1-B3, A1 grounded \\
\hline
\end{tabular}

\section{Results}

\subsection{Ferritin morphology on Si surface}

Figure 2a shows the a single layer of ferritin formed on the Si substrate. A continuous layer can be observed on the Si surface. Line profile (figure $2 \mathrm{f}$ ) indicated that the size of ferritin is about $7 \mathrm{~nm}$ height, which is similar to the actual size of ferritin as reported [2-5, 7-8, 13-14]. However, the lateral size of ferritin shown in figure $2 \mathrm{f}$ is $\sim 50 \mathrm{~nm}$, possibly due to the widening effect of the AFM probe. Figure $2 \mathrm{~b}-2 \mathrm{e}$ show that DMFS were able to be formed using the layer-by-layer technique described in [14]. The ferritin in these structures appears to be fairly continuous and compact. The same technique was used for fabricating each of the test dies. Although it was not possible to perform lateral current tests on those devices, c-AFM was used to measure the I-V properties of these ferritin on Si substrate. The results were shown in Figure 3, and are consistent with previously reported nonlinear I-V properties of ferritin using c-AFM [14]. 
(a)

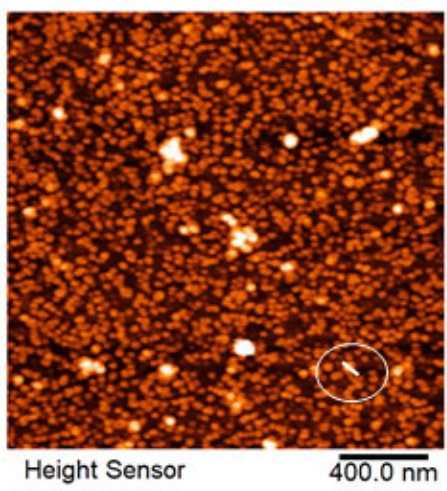

(c)

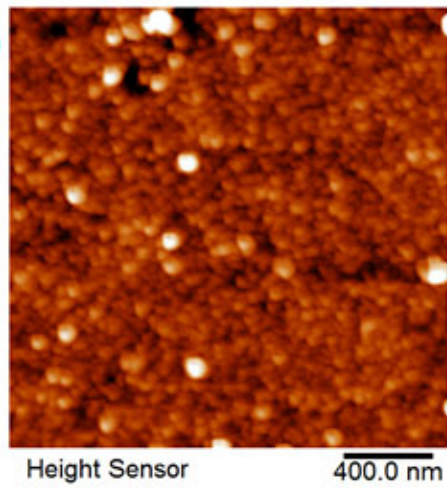

(e)

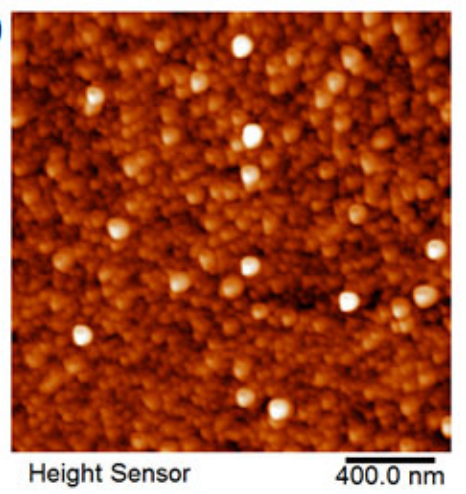

(b)
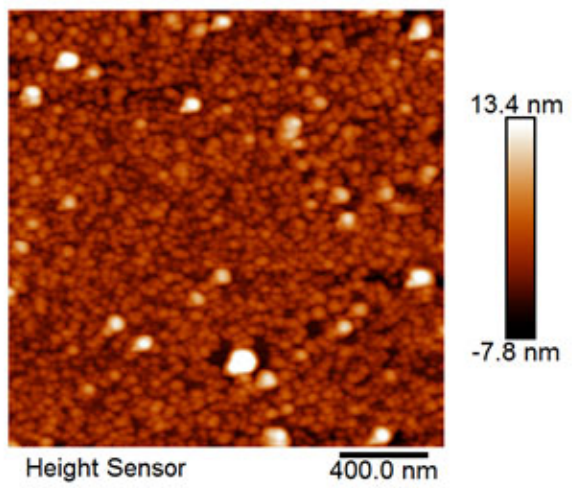

(d)
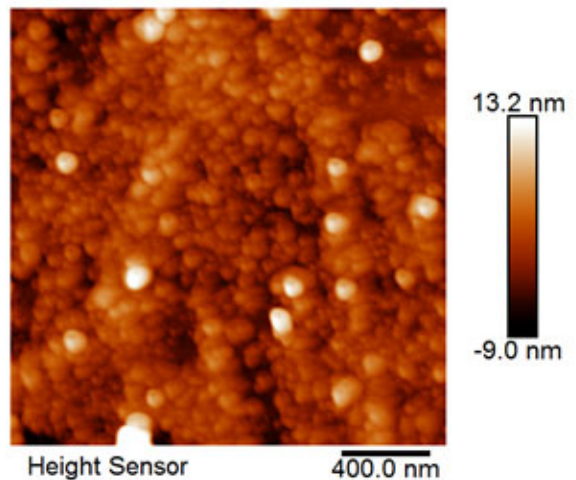

$-6.4 \mathrm{~nm}$

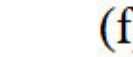

(f)
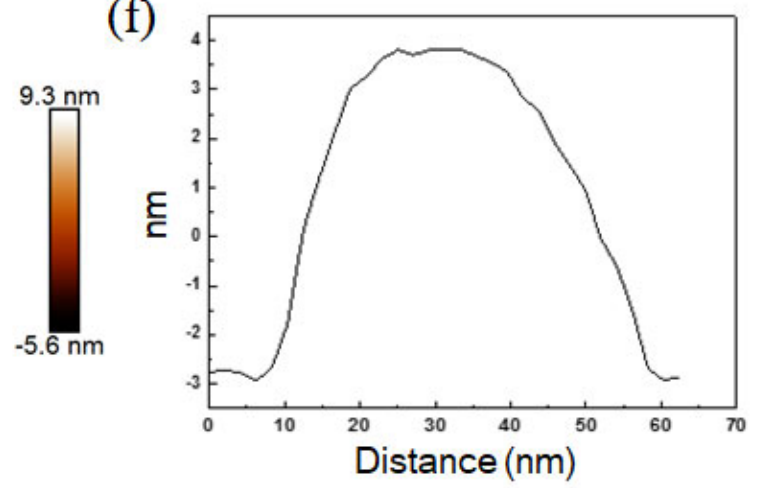

Figure 2. Ferritin grows on substrate. (a) one layer; (b) two layers; (c) three layers; (d) four layers; (5) five layers; (f) line profile of the ferritin as shown in (a) indicated in the circle. 


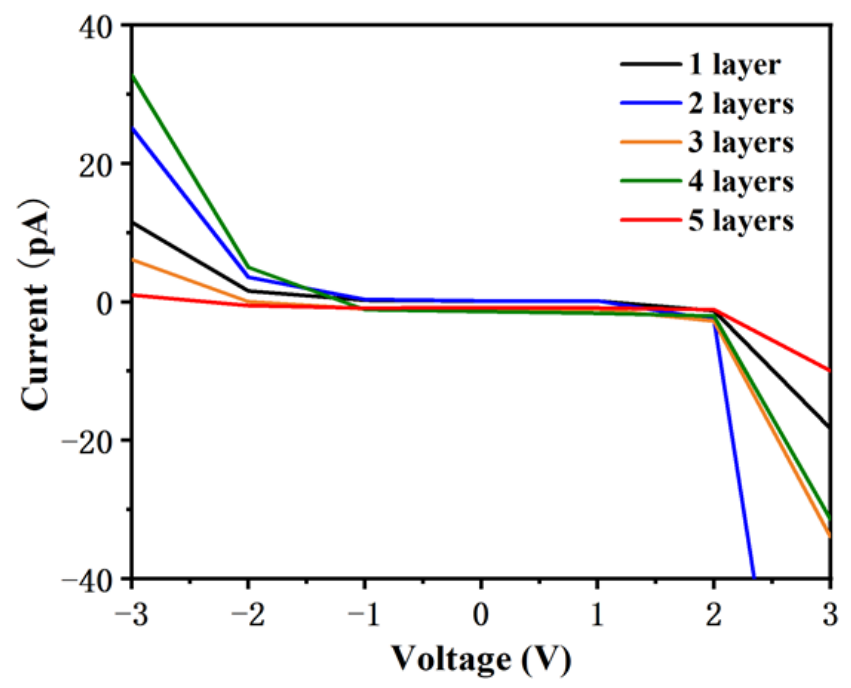

Figure 3. I-V properties of ferritin grows on Si substrate measured by c-AFM

\subsection{Ferritin on Si surface measured by c-AFM}

It was determined from the c-AFM tests that the surface topography of one layer of ferritin is not uniform, and some voids can be found on the surface as shown in figure $2 \mathrm{a}$. Nevertheless, electrical continuities can still be observed, see figure 3. This was not previously reported in [14], which lacked this type of detail and instead reported I-V measurements at individual points. The DMFS formed by the layer-by-layer deposition technique were shown in figure $2 b$-e. The c-AFM current measurements obtained from these layers also show an increase in current at voltages greater than $2.0 \mathrm{~V}$ and less than $-2.0 \mathrm{~V}$ for all the devices. For 5 layers of ferritin, there is a decrease of current which might be due to the thickness of ferritin has reach the peak-current value because of insulation.

While the discontinuities did not appear to form large areas without any ferritin, they were still significant enough to raise concerns regarding whether they would prevent the formation of DMFS that were capable of electron transport. While the layer-by-layer technique subsequently appeared to be partially effective for the purpose of these tests and to provide areas where DMFS were formed, those structures do not appear to be sufficiently continuous for use in manufacturing devices when made using the process discussed in [14].

\subsection{Ferritin on Si surface measured by Keithley measurements}


Tests on dies without any ferritin layers resulted in currents that were less than $100 \mathrm{pA}$. One of the two die configurations with interdigitated electrodes that was tested is shown below in Fig. 3(a), and is referred to as model 1. This die had a configuration similar to the die tested in [14], except for the modification to create 3 electrodes B1, B2 and B3:

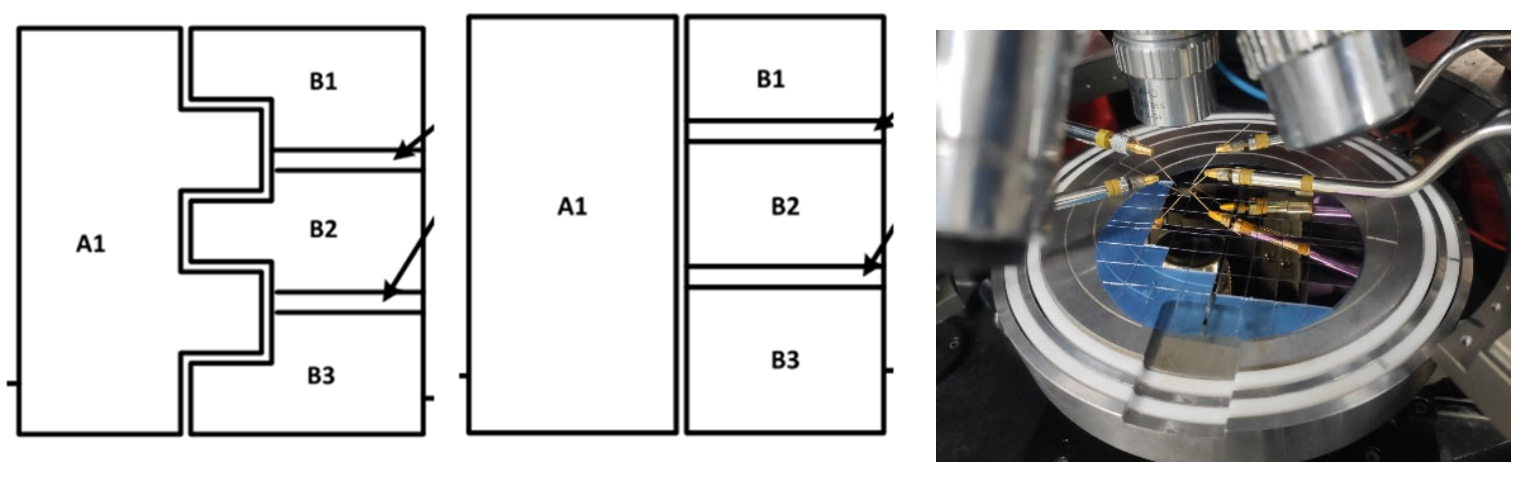

Fig. 3(a)-Model 1; 3(b) Model 2, c) picture of test configuration

A second test die was also made that did not use interdigitated electrodes. The design is shown in Fig. 3(b). The electrodes A1, B1, B2 and B3 were formed from gold on a silicon oxide substrate. Three different gaps between electrode A1 and electrodes B1, B2 and B3 were used (20, 40 and 80 microns). The electrodes B1, B2 and B3 were isolated from each other with silicon dioxide, as shown by the arrows.

The test equipment configuration is shown in Fig. 3(c), and included shielded leads for contacts to the test die to reduce the effects of noise caused by environmental EFI, and a separate ground connection to reduce DC offset or other grounding effects. Voltage and current measurements were derived from the leads.

A first set of tests on a model 2 die with no ferritin resulted in low current levels. For a first configuration with electrodes B1, B2 and B3 connected to ground and voltage applied to electrode A, the I/V curve shown in Fig. 5 in the Supporting Information was obtained. This current appears to be due to a DC calibration offset, because there is no variation in current and also because there is $450 \mathrm{pA}$ measured at 0 volts. It is noted that performing measurements of currents at this level is difficult, and that in this case the discrepancy can be explained. Other DC offset measurements were also recorded, and it was possible to account for those DC offsets in the small number of measurements where they were present. 
For a second configuration, electrodes B2 and B3 were left ungrounded (float) to simulate a high impedance path to ground, electrode B1 was grounded, and voltage was applied to electrode A1. The measured I/V response is shown in Fig. 6 in the Supporting Information. These measurements appear to be mostly noise below $30 \mathrm{pA}$, which indicates that any current that was flowing between the electrodes with no ferritin deposited was below the sensitivity of the test equipment. As previously mentioned, measuring current at the level of picoamperes is difficult, and a low level of current between electrodes of the bare dies is expected and consistent with the results reported in [14].

For a third test configuration, electrodes B1 and B3 were left ungrounded, electrode B2 was grounded, and voltage was applied to electrode A1. The measured I/V response is shown in Fig. 7 in the Supporting Information. These measurements appear to be mostly noise below 100 $\mathrm{pA}$, which again indicates that any current that was flowing between the electrodes with no ferritin deposited was below the sensitivity of the test equipment.

For a fourth test configuration, electrodes B1 and B2 were left ungrounded, electrode B3 was grounded, and voltage was applied to electrode A1. The measured I/V response is shown in Fig. 8 in the Supporting Information. These measurements appear to be mostly noise below $30 \mathrm{pA}$, which indicates that any current that was flowing between the electrodes with no ferritin deposited was below the sensitivity of the test equipment.

For a fifth configuration, electrode A1 was connected to ground and electrodes B1, B2 and B3 connected to the applied voltage. The measured I/V response is shown in Fig. 9 in the Supporting Information. These results mirror the results from the first test configuration, and also appear reflect a DC offset, because there is no variation in current and there is $-550 \mathrm{pA}$ measured at 0 volts. Thus, disregarding the data from the first and fifth test configurations that indicates a DC offset at the current measuring equipment, it appears that the dies without ferritin conduct current below the $100 \mathrm{pA}$ sensitivity of the test equipment. Tests were performed on 19 additional blank dies with similar results.

Tests were then performed on different dies with differing numbers of layers and different gap spacings, but many of those tests yielded current measurements of low-level noise that was below the sensitivity of the test equipment, or other unreliable results that indicated possible damage. Table 1 in the Supporting Information summarizes these test results, and identifies tests where currents of greater than 1 nanoampere with no apparent high frequency noise components 
were measured. As can be seen in that table, 8 of 36 tests produced results that were above what appeared to be the sensitivity of the test equipment, or which were not otherwise the results of possible contamination or damage (dies were damaged during a sonic cleaning process that was peformed for some dies, which were reused). Thus, it is concluded that while the layer-by-layer deposition of ferritin as disclosed in [14] can produce some useful results, that it is not a reliable process for producing devices with repeatable electrical characteristics. Several of the 8 tests that yielded measureable results are discussed further below.

Several important observations can be made from these results. First, despite the fact that the layer-by-layer ferritin deposition resulted in substantial amounts of ferritin being deposited, many of the tests resulted in measured currents between electrodes of less than $100 \mathrm{pA}$ and essentially equal to the current measured for bare dies without ferritin. As such, those tests establish that merely depositing ferritin in a multilayer structure using the layer-by-layer technique does not cause a measurable change in the measured current. This makes sense, given that the ferritin protein complexes themselves are only $12 \mathrm{~nm}$ in diameter and have almost no current measured for applied volatges of less than $0.5 \mathrm{~V}$.

The tests that resulted in currents greater than $100 \mathrm{pA}$ appear to be due to the formation of DMFS that are behaving in a non-classical manner (i.e. with non-linear I/V response) that is consistent with electron transport through DMFS, similar to what was measured in [14]. However, it was not possible to precisely determine the relationship between variations in gap size or the number of layers of the DMFS. Even though these results generally indicated an increase in current with decreasing gap size and an increasing number of layers, they did not allow for conclusions to be made regarding the mathematical relationship of current as a function of parametric variations on the number of layers and the spacings between electrodes to be made.

The results of specific tests are discussed in greater detail below.

\section{3 (A) $80 \mu \mathrm{m}$ gap, 4 layers (model 2)}

The first test to be discussed was on a type 2 die with 4 layers of ferritin and an 80 micron gap. While the logic/objective of the different gaps and layers was to determine the mathematical relationship associated with increasing layers and increasing gaps, the results of these tests were unable to accomplish that objective. However, the 8 tests that were effective at generating currents greater than $100 \mathrm{pA}$ with a smooth nonlinear I/V response were able to accomplish other objectives: 1) establishing that layer-by-layer ferritin deposition is capable of forming DMFS, and 
2) that the DMFS exhibit highly correlated electron transport behavior, as shown by pathdependent electron transport behavior such as diode-like switching.

For test configuration 1, the I/V curve of Fig. 4(a) was obtained.

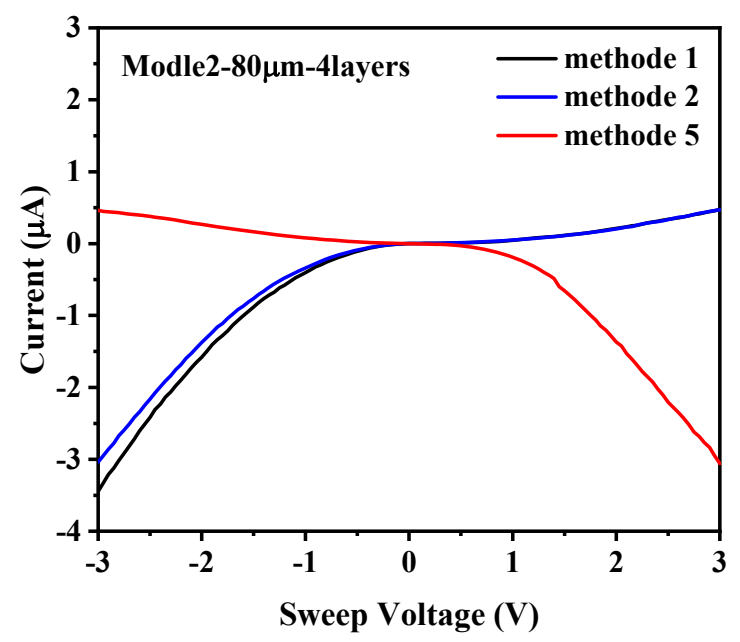

Fig. 4 - I/V curves for $80 \mu \mathrm{m}$ gap, 4 layer test specimen, a) voltage applied to A1, B1-B3 grounded (configuration 1); b) voltage applied to A1, B1 grounded, B2 and B3 float

(configuration 2); c) voltage applied to B1-B3, A1 grounded (configuration 5).

These results show a substantial current level of $-3 \mu \mathrm{A}$ at $-3 \mathrm{~V}$, and about $500 \mathrm{nA}$ at $3 \mathrm{~V}$, with a log-linear/non-linear increase in current with voltage. This nonlinear I/V behavior is consistent with modelled electron transport through QD arrays, as opposed to a linear I/V response that would be expected for classical conduction.

For test configuration 2 (electrode A1 to electrode B1, with electrodes B2 and B3 floating), the results are shown in Fig. 4(b). It is noted that these results are essentially identical to the results from configuration 1 . The fact that the I/V response for the single current path through A1 and B1 is the same as the I/V response for the current path through $\mathrm{A}$ and $\mathrm{B} 1, \mathrm{~B} 2$ and $\mathrm{B} 3$ in parallel indocates that there was only a single DMFS between A1 and B1, and no DMFS between A1 and $\mathrm{B} 2$ or $\mathrm{A} 1$ and $\mathrm{B} 3$.

For configurations 2 and 3, relatively low level noise was measured, which also appears to indicate that there was no DMFS that formed an electron transport connection between electrodes A1 and either of electrodes B2 and B3, see Fig. 9 in the Supporting Information. However, it is noted that the magnitude of the current measured between electrodes A1 and B2 was slighty higher 
(200 pA) than what appears to be the sensitivity of the equipment (100 pA), which may reflect a small amount of electron transport.

For configuration 5, the measured current was similar to configurations 1 and 2, see Fig. 4(c). The "mirror image" effect of measuring negative currents at positive applied voltages was due to a reversed current measurement direction relative to the applied voltage (i.e. current measured is flowing out to the ground lead instead of into the die). These test results show a loglinear/non-linear I/V response, which is consistent with the models in [6]. The reason for the difference between higher levels of measured negative currents at -3 volts and lower levels of positive currents at +3 volts is unclear, but may be due to differences in electron and hole mobility in the DMFS, non-uniform DMFS between electrodes A1 and B2, DMFS between electrodes B1, $\mathrm{B} 2$ and $\mathrm{B} 3$ that exclude electrode A1 or other variations.

\section{3(B) $20 \mu \mathrm{m}$ gap, 3 layers and retest (model 1)}

A second test that yielded results greater than the equipment sensitivity was a model 1 die with 3 layers of ferritin and a $20 \mu \mathrm{m}$ gap. For the initial test, the configuration 1 test yielded $-9 \mathrm{nA}$ at $-3 \mathrm{~V}$ and $4 \mathrm{nA}$ at $+3 \mathrm{~V}$, with a $\log$-linear/non-linear $\mathrm{I} / \mathrm{V}$ response curve for negative currents, but a different non-linear response for positive currents, see Fig. 5(a).
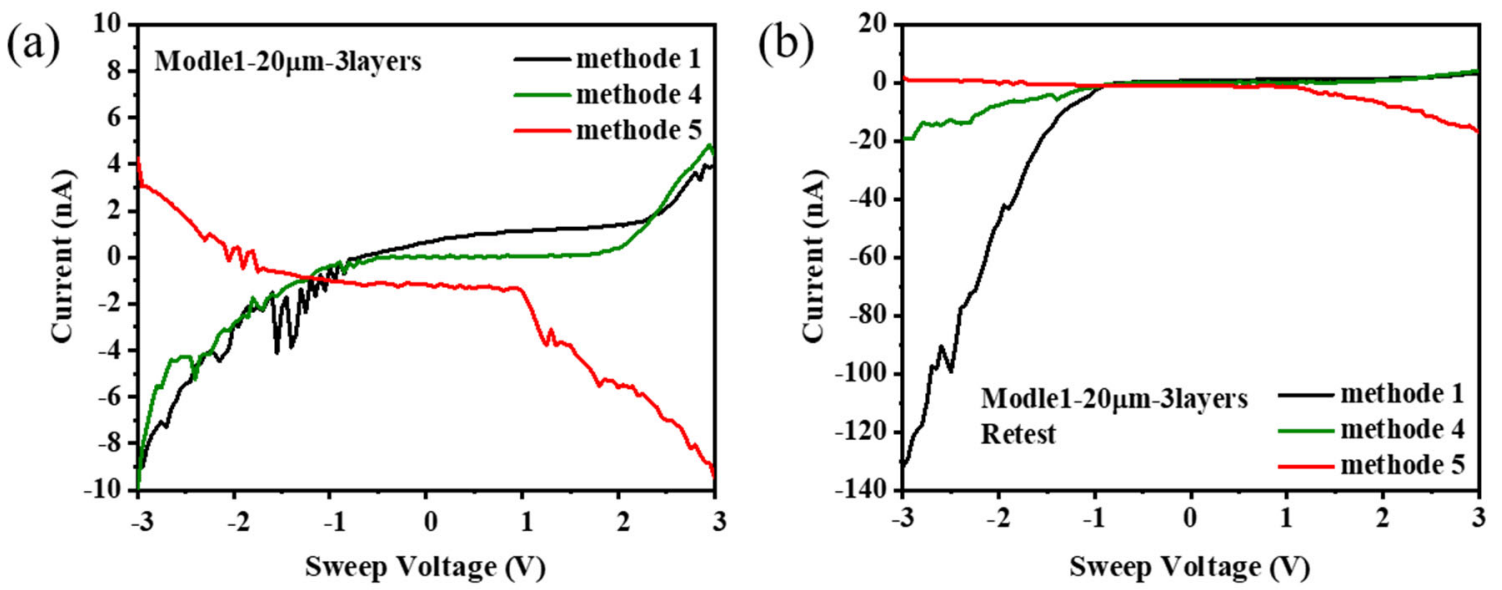

Fig. 5(a) - I/V curves for $20 \mu \mathrm{m}$ gap, 3 layers, voltage applied to a) A1, B-1B3 grounded (configuration 1); b) A1, B3 grounded, B1 and B2 float (configuration 4); c) B1-B3, A1 grounded (configuration 5).

Fig. 5(b) -Retest I/V curve for $20 \mu \mathrm{m}$ gap, 3 layers, voltage applied to a) A1, B1-B3 grounded (configuration 1); b) A1, B3 grounded, B1 and B2 float (configuration 4); c) B1-B3, A1 grounded (configuration 5). 
Configuration 2 yielded noise below $100 \mathrm{pA}$, but configuration 3 yielded noise that was slightly higher, which may indicate some weak ferritin layer connections between B2 and A1 for this die, see Fig. 10 in the Supporting Information. The fourth configuration also yielded - $9 \mathrm{nA}$ at $-3 \mathrm{~V}$ and $4 \mathrm{nA}$ at $+3 \mathrm{~V}$, with a $\log$-linear/non-linear $\mathrm{I} / \mathrm{V}$ response behavior on the negative current side of the curve, but the different non-linear response for positive currents, see Fig. 5(b).

Configuration 5 yielded $-9 \mathrm{nA}$ at $3 \mathrm{~V}$ and $4 \mathrm{nA}$ at $-3 \mathrm{~V}$, with a $\mathrm{I} / \mathrm{V}$ response curve that is nonlinear, see Fig. 5(c). Based on these results, it appears that there was only a single good ferritin layer connection between A1 and B3, but that there may have been a second weaker DMFS connection between $\mathrm{A} 1$ and $\mathrm{B} 3$, which could have resulted in a more complex current distribution, capacitive charging effects or other electrical behavior that had an impact on the I/V response.

This die was then stored for several weeks in an inert, dry nitrogen atmosphere at 4 degrees celsius, and then retested, At the retest, the I/V behaviour was different. For configuration 1, the results were -90 to $-130 \mathrm{nA}$ at $-3 \mathrm{~V}$ and less than $5 \mathrm{nA}$ at $3 \mathrm{~V}$ for configuration 1 in the initial test, see Fig. 5(b).

The I/V behavior for configurations 2 and 3 also indicated that a change occurred in the $\mathrm{I} / \mathrm{V}$ behavior of the A1-B1 connection, with an increase from a maximum noise level of $80 \mathrm{pA}$ to a maximum noise level of 180 pA, see Fig. 11 in the Supporting Information.

For configuration 4, $-18 \mathrm{nA}$ was measured at $-3 \mathrm{~V}$ and $4 \mathrm{nA}$ at $3 \mathrm{~V}$, with a log-linear/nonlinear response for the negative currents but a different non-linear response for the positive currents. For configuration 5, the reverse of the I/V measurements for configuration 4 was measured, as opposed to a reverse of configuration 1.

\section{3(C) $20 \mu \mathrm{m}$ gap, 4 layers (model 1)}

For tests on a model 1 die with a $20 \mu \mathrm{m}$ gap and 4 layers of ferritin, the current increased to -30 nanoamperes at 4 layers with a $20 \mu \mathrm{m}$ gap, which is consistent with an expected improvement in current with additional layers, see Fig. 6(a). 
(a)

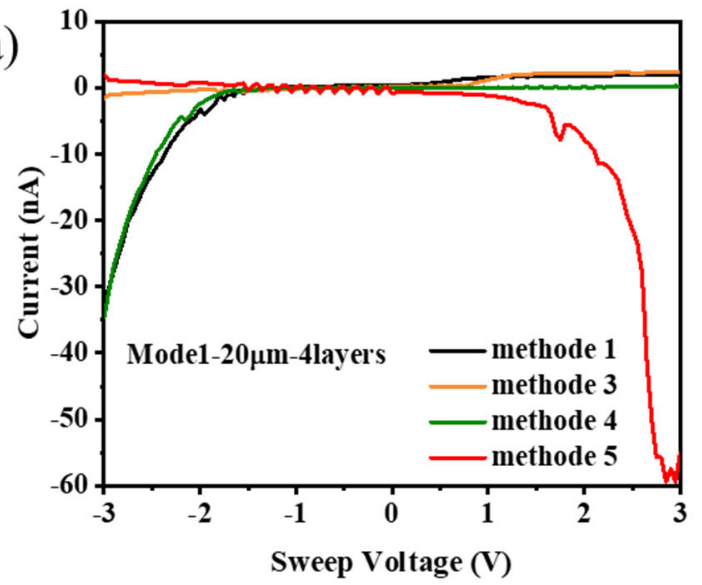

(b)

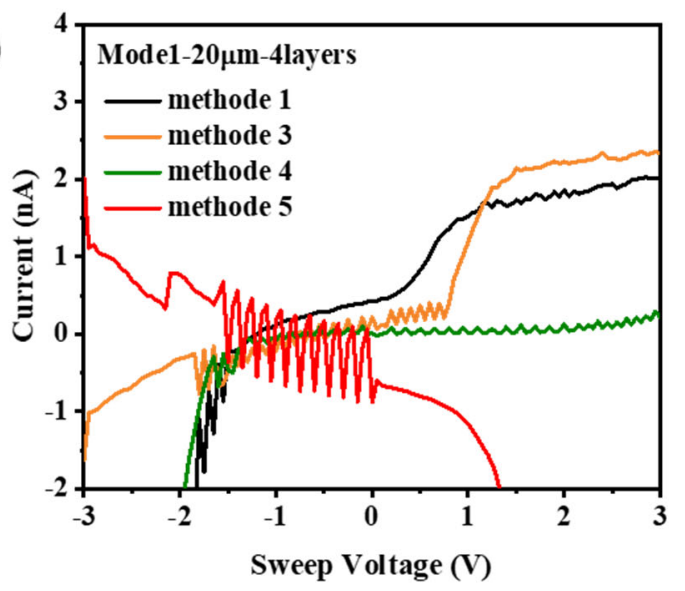

Fig. 6 - I/V curves for $20 \mu \mathrm{m}$ gap, 4 layers, voltage applied to: a) A1, B1-B3 grounded (configuration 1); b) A1, B2 grounded, B1, B3 float (configuration 2); c) A1, B3 grounded, B1, B2 float (configuration 4); d) B1-B3, A1 grounded (configuration 5).

For configuration 2, low-level noise was measured, consistent with other measurements of current below the sensitivity of the test equipment. For configuration 3, current in excess of noise levels was measured, with a highly nonlinear I/V response, see Fig. 7(b). For configuration 4, the I/V response was similar to configuration 1, see Fig. 7(c). These results also provide evidence of an impedance-based switching effect, similar to those obtained for the tests in Section II.B.

\section{3(D) Model 1, 5 layers $40 \mu \mathrm{m}$}

The results of I/V tests for configuration 1 of a model 1 die with 5 layers of ferritin and a $40 \mu \mathrm{m}$ gap were $-3 \mu \mathrm{A}$ at $-3 \mathrm{~V}$ and $2 \mu \mathrm{A}$ at $3 \mathrm{~V}$, see Fig. 8(a).

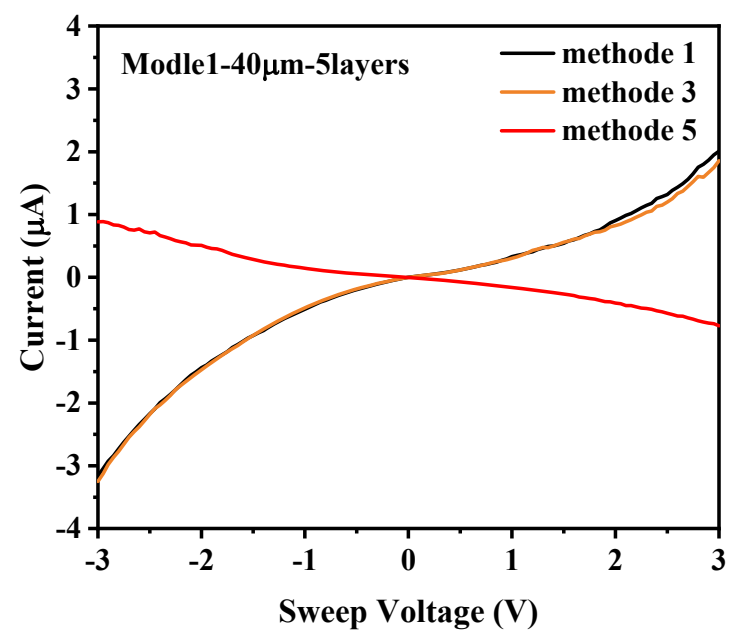


Fig. 7 - a) I/V curve for $40 \mu \mathrm{m}$ gap, 5 layers, voltage applied to: a) A1, B1-B3 grounded (configuration 1); b) B1-B3, A1 grounded (configuration 5).

The results for tests on configuration 2 were low levels of noise, consistent with other measurements below the sensitivity of the test equipment for different configurations. For configuration 4, there was a high level current transient at -1 V that may be indicative of a DMFS configuration that resulted in at least some electron transport, see Fig. 12 in the Supporting Information.

Configuration 3 resulted in the same $\mathrm{I} / \mathrm{V}$ behavior as configuration 1 . Configuration 5 resulted in a I/V curve that ranged from $900 \mathrm{nA}$ at $-3 \mathrm{~V}$ to $-700 \mathrm{nA}$ at $3 \mathrm{~V}$, in a log-linear/nonlinear distribution, see Fig. 8(b).

\section{Discussion}

The observed I/V behavior of the different configurations of dies and layers provides evidence that layer-by-layer ferritin deposition is able to form DMFS with sufficient order to support coherent electron transport between parallel electrodes, and also demonstrates that these currents are not an artifact of the ferritin itself, but rather of the ferritin in the multilayered configurations. In particular, when the degree of disorder is too great, then the result is that the currents measured are consistent with the currents measured when there is no ferritin. This is not surprising, because the layers of ferritin are very thin (approximately $12 \mathrm{~nm}$ per layer), plus the individual ferritin cores exhibit near-zero conductivity at voltages lower than $0.5 \mathrm{~V}$. When thousands of ferritin cores are placed in series and a voltage of $3 \mathrm{~V}$ or less is applied, conventional current flow would not be expected, in light of the measured I/V response of individual ferritin cores.

However, the substantially higher levels of current that were measured in roughly $25 \%$ of the tested dies demonstrates that the layer-by-layer deposition technique is capable of providing at least small areas with DMFS that form a continuous path between two or more electrodes. The electrical discontinuities seen in the DMFS from the c-AFM data establish that the layer-by-layer technique does not create an electrically homogenous structure, and these observed electrical discontinuities in the DMFS appear to be sufficient to prevent conduction between electrodes in most dies. However, the observed I/V response at levels of 1 nanoampere or greater demonstrates 
that electron transport is occurring at levels at least one order of magnitude greater than the I/V response of $100 \mathrm{pA}$ or less, which is consistent with the current measured for bare dies.

Furthermore, these tests demonstrate that the electron transport was highly correlated, consistent with the behavior of electrons in QD arrays. In particular, electron blocking was observed for multiple current paths through DMFS that have the same impedance, where electrons were able to flow when only a single path was available. This would be expected, because highly correlated electrons will try to follow the same path, which would not be possible when two or more paths are present.

For example, in Section 3.3(A), the I/V data shown in Figure 4 is generally consistent with highly correlated electron behavior in QD arrays. The same current behavior was observed for current flow between electrode A1 and electrodes B1-B3 in parallel and grounded (configuration 1) as was observed for electrode B1 alone grounded with electrodes B2 and B3 floating (configuration 2). In contrast, only low levels of current were measured for electrode B2 grounded with electrodes B1 and B3 floating (configuration 3) and for electrode B3 grounded with electrodes B 1 and B2 floating (configuration 4). Likewise, in configuration 5, the same I/V behavior was observed as for configurations 1 and 2 . In addition, the $\mathrm{I} / \mathrm{V}$ profile for negative voltages is different from the I/V profile for positive voltages, which indicates that electron transport is path-dependent and not a function of classical impedance.

For section 3.3(B), the data shown in Figures 5 and 6 is consistent with this analysis. For the original test configuration, there was an apparent DC offset for the configuration data, with a log-linear/nonlinear I/V response for negative applied voltage and a zero current response up to 2 volts, after which the current increased non-linearly. For configuration 2, the current was below the noise/measurement sensitivity level of the current meter. For configuration 3, the noise level was slightly higher, which may indicate some low levels of current conduction through DMFS. For configuration 4 , the I/V data was similar to the configuration 1 data without a DC offset, and for configuration 5 , the $\mathrm{I} / \mathrm{V}$ data was also similar to configuration 1 , with a reversed direction reflecting the relative voltage measurement convention. The $\mathrm{I} / \mathrm{V}$ behavior indicates that positive currents were blocked up to $2 \mathrm{~V}$, but that negative currents were able to flow in the retest configuration.

It would be expected that all current would be blocked in both B1-B3 parallel connections of test configurations 1 and 5 (with positive and negative currents), not just effectively half of the 
I/V data for those configurations (where current flows into electrode A1 and electrons flow from electrode A1 to electrodes B1 through B3). The non-symmetric I/V behavior indicates that electrons travelling from electrode A1 to electrodes B1-B3 see a different path than electrons travelling from electrodes B1-B3 in parallel to electrode A1, such as due to different DMFS. Structures that connect two or more of electrodes B1-B3 but which do not connect to electrode A1 would present electrons at electrodes B1-B3 with multiple paths, where the electrons are at the same potential and are exposed to the same electric field vector, as shown below in Fig. 9.

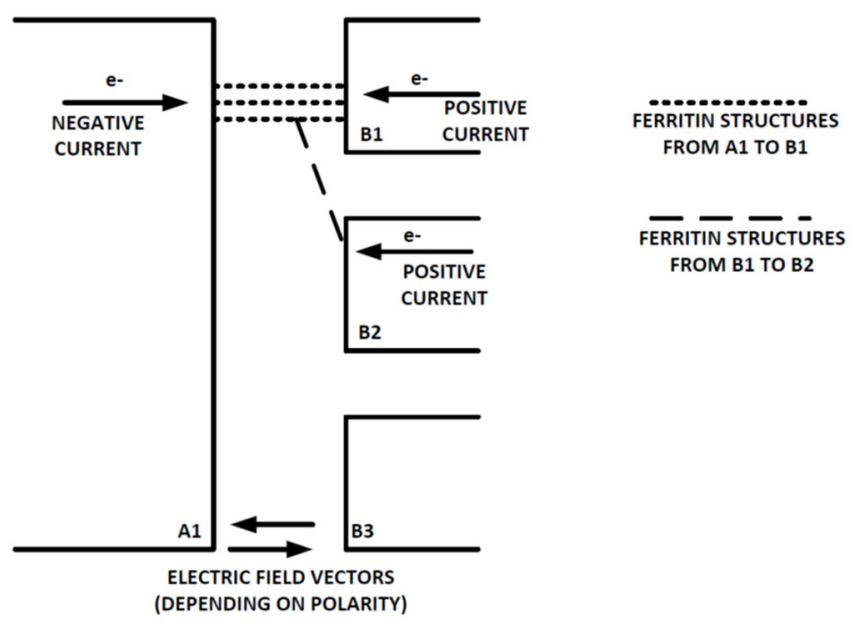

Fig. 9 - Electron Transport Structures

Electrons traveling from electrodes $\mathrm{B} 1$ and $\mathrm{B} 2$ on the right to electrode $\mathrm{A} 1$ on the left in this figure would see different DMFS with a common connection, but each ferritin core would nonetheless be at the same potential and would be exposed to the same electric field vector, at least at low voltage levels. This first configuration would generate highly correlated electrons, but they would be unable to tunnel from electrodes B1 and B2 because those are different physical paths. In contrast, electrons traveling from electrode $\mathrm{A} 1$ on the left to electrodes $\mathrm{B} 1$ and $\mathrm{B} 2$ on the right would see a different DMFS, where each ferritin core is at the same potential and is exposed to the same electric field vector. This second configuration would also generate highly correlated electrons, which would be able to tunnel from electrode A1 to electrode B1. The DMFS between electrodes B1 and B2 would not interfere with tunneling in this configuration.

Furthermore, in the first configuration, the electric field vector at electrode B1 would start to diverge from the electric field vector at electrode B2 as the applied voltage at electrode A1 is increased from 0 to 3 volts. Thus, at low voltages, the different structures would block electron 
transport from right to left, but when the voltage reaches a high enough level to cause the electric fields to diverge and block highly correlated electron behavior, current would be able to flow through the dominant B1-A1 DMFS. It is not possible to determine the exact configuration of the ferritin layer structures, or to easily model the behavior of such structures even it was known, but subsequent testing with more uniform structures should be able to eliminate this unbalanced I/V response.

For the restest, it is unclear why the current decreased from -90 to $-130 \mathrm{nA}$ at $-3 \mathrm{~V}$ (the reverse of configuration 1) to $-14 \mathrm{nA}$ at $-3 \mathrm{~V}$ (the reverse of configuration 4). It is possible that ferritin layer connections between electrodes B1-B3 cause capacitive charging effects to occur during the tests for configurations 2-4 that had an impact on the tests for configuration 5, but it also cannot be conclusively ruled out that probe movement during test configuration charges or other variables had an effect.

For section 3.3(C), the data shown in Fig. 7 are also consistent with the existence of multiple ferritin layer connections between electrodes A1 and B2, or possibly ferritin layer connections between B2 and either of B1 or B3. Current blocking occurred for configurations 1, 4 and 5 , but no current was measured for configuration 2 and a highly non-linear I/V curve was measured for configuration 3. This indicates that both electrodes B2 and B3 had some DMFS connections to electrode A1, but that the connection between B2 and A1 was weaker, and that the structure between B2 and B3 was configured so as to block electron transport up to 3 volts when B3 was grounded but only up to 1 volt when B2 was grounded.

For section 3.3(D), the data shown in Fig. 8 are unusual because there is a change from the measurements for configuration 1, and there is also not a greater negative current than positive current. While it is unclear why there was a difference between the results for configurations 1 and 5, these results could be explained by a single DMFS connection between electrodes A1 and B2, such that no switching effect occurred. However, the transient at $-1 \mathrm{~V}$ for test configuration 4 could have resulted in a structural change in a ferritin layer, capacitive charging or some other effect that caused the $\mathrm{I} / \mathrm{V}$ response to change.

\section{Conclusion}

While the layer by layer approach to forming DMFS provides inconsistent results, it is sufficient to provide evidence of quantum mechanical electron transport in such structures. If such 
electron transport was not occurring, then the I/V measurements should be at the $100 \mathrm{pA}$ level or lower, as shown by many test results where such levels were observed. The fact that at least some tests were able to produce non-linear currents at up to 5 orders of magnitude greater than the current capacity of bare dies is consistent with electron transport through disordered QD arrays, as is the difference in I/V behavior as a function of the direction of electron travel. However, these tests were unable to control layer configurations sufficiently to perform parametric analysis of difference in layers and distance, and further testing with more control over the DMFS would be needed, either by improving the layer-by-layer deposition technique to prevent the formation of the electrical discontinuities reported here for the first time, or by using micromanipulators or other more expensive equipment and time-intensive procedures.

\section{Acknowledgement:}

Cai, Yunbo and Minjing thanks financial supports from the Natural Science Foundation of Zhejiang and Taizhou (LTY20B030001) and private fund from Chris Rourk.

\section{Reference}

[1] H.C. Chung, W.H. Chu, C.P. Liu, Electron transport through individual Ge self-assembled quantum dots on Si, Applied Physics Letters, 89 (2006) 3.

[2] D.N. Axford, J.J. Davis, Electron flux through apo- and holoferritin, Nanotechnology, 18 (2007) 7.

[3] J. Kolay, S. Bera, T. Rakshit, R. Mukhopadhyay, Negative Differential Resistance Behavior of the Iron Storage Protein Ferritin, Langmuir, 34 (2018) 3126-3135.

[4] D.G. Xu, G.D. Watt, J.N. Harb, R.C. Davis, Electrical conductivity of ferritin proteins by conductive AFM, Nano Letters, 5 (2005) 571-577.

[5] T. Rakshit, S. Banerjee, R. Mukhopadhyay, Near-Metallic Behavior of Warm Holoferritin Molecules on a Gold(111) Surface, Langmuir, 26 (2010) 16005-16012.

[6] Middleton Alan, J. Shantenu, Effects of Disorder on Electron Transport in Arrays of Quantum Dots, Physics, 184 (2005).

[7] Y.-H. Pan, K. Sader, J.J. Powell, A. Bleloch, M. Gass, J. Trinick, A. Warley, A. Li, R. Brydson, A. Brown, 3D morphology of the human hepatic ferritin mineral core: New evidence for a subunit structure revealed by single particle analysis of HAADF-STEM images, Journal of Structural Biology, 166 (2009) 22-31.

[8] U. Carmona, L. Li, L.B. Zhang, M. Knez, Ferritin light-chain subunits: key elements for the electron transfer across the protein cage, Chemical Communications, 50 (2014) 15358-15361. [9] J.A. Soltis, A.M. Schwartzberg, P. Zarzycki, R.L. Penn, K.M. Rosso, B. Gilbert, Electron Mobility and Trapping in Ferrihydrite Nanoparticles, Acs Earth and Space Chemistry, 1 (2017) 216-226. 
[10] M. Naito, K. Iwahori, A. Miura, M. Yamane, I. Yamashita, Circularly Polarized Luminescent CdS Quantum Dots Prepared in a Protein Nanocage, Angewandte ChemieInternational Edition, 49 (2010) 7006-7009.

[11] C. Kulkarni, A.K. Mondal, T.K. Das, G. Grinbom, F. Tassinari, M.F.J. Mabesoone, E.W. Meijer, R. Naaman, Highly Efficient and Tunable Filtering of Electrons' Spin by Supramolecular Chirality of Nanofiber-Based Materials, Advanced Materials, 32 (2020).

[12] L. Wang, Z.L. Wang, Advances in piezotronic transistors and piezotronics, Nano Today, 37 (2021).

[13] S.K. Karuppannan, R.R. Pasula, T.S. Herng, J. Ding, X. Chi, E.D. Barco, S. Roche, X. Yu, N. Yakovlev, S. Lim, C.A. Nijhuis, Room-temperature tunnel magnetoresistance across biomolecular tunnel junctions based on ferritin, Journal of Physics: Materials, 4 (2021). [14] S. Bera, J. Kolay, P. Pramanik, A. Bhattacharyya, R. Mukhopadhyay, Long-range solidstate electron transport through ferritin multilayers, Journal of Materials Chemistry C, 7 (2019) 9038-9048.

[15] C. Herrmann, G.C. Solomon, M.A. Ratner, Designing organic spin filters in the coherent tunneling regime, Journal of Chemical Physics, 134 (2011) 9.

[16] D. Sala, S. Ciambellotti, A. Giachetti, P. Turano, A. Rosato, Investigation of the Iron(II) Release Mechanism of Human H-Ferritin as a Function of $\mathrm{pH}$, Journal of Chemical Information and Modeling, 57 (2017) 2112-2118.

[17] G.B. Martins, C.A. Busser, K.A. Al-Hassanieh, E.V. Anda, A. Moreo, E. Dagotto, Transport properties of strongly correlated electrons in quantum dots studied with a simple circuit model, Physical Review Letters, 96 (2006) 4.

[18] S. Kalliakos, M. Rontani, V. Pellegrini, C.P. Garcia, A. Pinczuk, G. Goldoni, E. Molinari, L.N. Pfeiffer, K.W. West, A molecular state of correlated electrons in a quantum dot, Nature Physics, 4 (2008) 467-471.

[19] M.H. He, Y.H. Li, J.Q. Cai, Y. Liu, K. Watanabe, T. Taniguchi, X.D. Xu, M. Yankowitz, Symmetry breaking in twisted double bilayer graphene, Nature Physics, 17 (2021) 26-+. 